\title{
Integrated Molecular Profiling Studies to Characterize the Cellular Origins of High-Grade Serous Ovarian Cancer
}

Kate Lawrenson ${ }^{1,2 * \dagger}$, Marcos A. S. Fonseca ${ }^{3 *}$, Felipe Segato ${ }^{3 *}$, Janet M. Lee ${ }^{2}$, Rosario I. Corona ${ }^{1,2}$, Ji-Heui $\mathrm{Seo}^{4}$, Simon Coetzee ${ }^{2}$, Yvonne G. Lin ${ }^{5 \S}$, Tanja Pejovic ${ }^{6,7}$, Paulette Mhawech-Fauceglia $^{8}$, Ronny Drapkin ${ }^{9}$, Beth Y. Karlan ${ }^{1}$, Dennis J. Hazelett ${ }^{2}$, Matthew L. Freedman ${ }^{4}$, Simon A. Gayther ${ }^{2 \# t}$, Houtan Noushmehr ${ }^{3,10 \# t}$

\footnotetext{
1 Women's Cancer Program at the Samuel Oschin Comprehensive Cancer Institute, Cedars-Sinai Medical Center, 8700 Beverly Boulevard, Suite 290W, Los Angeles, CA, USA

${ }^{2}$ Center for Bioinformatics and Functional Genomics, Department of Biomedical Sciences, Cedars-Sinai Medical Center, Los Angeles, California, USA

3 Department of Genetics, Ribeirão Preto Medical School, University of São Paulo, 14049-900, Brazil

${ }^{4}$ Department of Medical Oncology, Dana-Farber Cancer Institute, Boston, MA, USA

${ }^{5}$ Division of Gynecologic Oncology, Department of Obstetrics-Gynecology, University of Southern California/Keck School of Medicine, 1450 Biggy Street, Los Angeles, California, USA

${ }^{6}$ Department of Obstetrics and Gynecology, Oregon Health and Science University, Portland, OR, USA

${ }^{7}$ Knight Cancer Institute, Oregon Health \& Science University, Portland, Oregon, USA

${ }^{8}$ Departments of Medicine and Pathology, University of Southern California/Keck School of Medicine, 1450 Biggy Street, Los Angeles, California, USA

9 Penn Ovarian Cancer Research Center, Department of Obstetrics and Gynecology, University of Pennsylvania, Philadelphia, PA, USA

10 Department of Neurosurgery, Henry Ford Hospital, Detroit, MI, USA

$\S$ current affiliation: Genentech, A member of the Roche family, 1 DNA Way, MS444A, South San Francisco, CA, USA
}

† Corresponding authors: Simon A. Gayther, Center for Bioinformatics and Functional Genomics, Department of Biomedical Sciences, Cedars-Sinai Medical Center, Los Angeles, California, USA; email: simon.gayther@cshs.org; phone: 310-423-2645. Houtan Noushmehr, Department of Neurosurgery, Henry Ford Hospital, Detroit, MI, USA; email: hnoushm1@hfhs.org; phone: 310-570-2362. Kate Lawrenson, Women's Cancer Program at the Samuel Oschin Comprehensive Cancer Institute, Cedars-Sinai Medical Center, 8700 Beverly Boulevard, Suite 290W, Los Angeles, CA, USA; email: kate.lawrenson@cshs.org, phone: 310-423-7935.

* equal contribution; * jointly directed the study

Running title: Integrated Molecular Profiling of Ovarian Cancer Origins

Keywords: high-grade serous ovarian cancer, ovarian surface epithelial cell, fallopian tube secretory epithelial cell, super-enhancers, SOX18

Conflicts of interest: The authors declare no potential conflicts of interest. 
bioRxiv preprint doi: https://doi.org/10.1101/330597; this version posted May 25, 2018. The copyright holder for this preprint (which was not certified by peer review) is the author/funder. All rights reserved. No reuse allowed without permission.

Word count: abstract 145 words, main text 2,620 words 


\section{Abstract}

Historically, high-grade serous ovarian cancers (HGSOCs) were thought to arise from ovarian surface epithelial cells (OSECs) but recent data implicate fallopian tube secretory epithelial cells (FTSECs) as the major precursor. We performed transcriptomic and epigenomic profiling to characterize molecular similarities between OSECs, FTSECs and HGSOCs. Transcriptomic signatures of FTSECs were preserved in most HGSOCs reinforcing FTSECs as the predominant cell-of-origin; though an OSEC-like signature was associated with increased chemosensitivity $\left(P_{a d j}=0.03\right)$ and was enriched in proliferative-type tumors, suggesting a dualistic model for HGSOC origins. More super-enhancers (SEs) were shared between FTSECs and HGSOCs than between OSECS and HGSOCs $\left(P<2.2 \times 10^{-16}\right)$. SOX18, ELF3 and EHF transcription factors (TFS) coincided with HGSOC SEs and represent putative novel drivers of tumor development. Our integrative analyses support a predominantly fallopian origin for HGSOCs and indicate tumorigenesis may be driven by different TFs according to cell-of-origin. 
Invasive epithelial ovarian cancers are a heterogenous group of tumors comprising several major histological subtypes: high-grade serous, low-grade serous, endometrioid, clear cell and mucinous. Highgrade serous ovarian cancer (HGSOC) is the most common subtype, comprising around two-thirds of all invasive cases. Our understanding of the cellular origins of HGSOC and key transcription factor networks deregulated during HGSOC development has been restricted by the lack of substantial molecular profiling data for the suggested precursor tissues, ovarian surface epithelial cells (OSECs) and fallopian tube secretory epithelial cells (FTSECs). Historically, HGSOCs were thought to arise from OSECs, an atypical epithelial cell type with mesothelial features and inherent phenotypic plasticity and heterogeneity ${ }^{1,2}$. However, examples of early-stage ovarian carcinoma arising from OSECs in vivo are scare, and the discovery of occult carcinomas in the fallopian tubes of $B R C A 1 / 2$ mutation carriers provided evidence to support an alternative hypothesis that the fallopian epithelium harbors the cell-of-origin for HGSOC ${ }^{3-7}$. Subsequent studies support a tubal origin for HGSOCs not associated with highly penetrant gene mutations, suggesting that HGSOCs arise from FTSECs in at least $50 \%$ of cases ${ }^{8-10}$. However, in a proportion of cases there remains no evidence of fallopian involvement, which raises the possibility that HGSOCs could originate from more than one tissue type. The large-scale molecular data sets required to fully evaluate the relationships of OSEC and FTSECs to HGSOC have been lacking, and so in the current study we established transcriptome-wide signatures in 188 normal cells representing these two putative origins of HGSOC. We used machine learning to quantify the similarities between OSECs and FTSECs to HGSOCs transcriptomes as a molecular approach to characterizing disease origins. In addition, we integrated transcriptomic data with epigenetic maps for these cell types, to characterize tissue-specific super-enhancer landscapes, and to identify putative transcription factors driving transcriptional deregulation during serous ovarian tumorigenesis.

\section{Results}

\section{Expression profiling of putative ovarian cancer precursor cells}

One approach to exploring cellular origins of cancer is to quantify similarities and differences between molecular signatures of tumors and the proposed tissues of origin ${ }^{11}$; based on the hypothesis that the molecular blueprint of the normal precursor cell is maintained in the developed tumor. We applied this approach to explore HGSOC origin by performing RNA-sequencing (RNA-seq) on an extensive series of normal cells isolated from the two major proposed precursor tissue types, ovarian surface epithelial cells (OSECs; $n=114$ ) and fallopian tube secretory epithelial cells (FTSECs; $n=74$ ). Cell samples were derived 
from 132 individuals, with both OSECs and FTSECs isolated from the same patient in 56 cases ${ }^{12,13}$

(Supplementary Table 1). For one sample, we replicated the RNA sequencing for quality control purposes; expression profiles from this samples were highly correlated (Pearson's correlation $r=0.98$ )

(Supplementary Fig. 1). We found no associations with experimental or epidemiological variables (where available), including sample preparation patient age or ethnicity (data not shown).

Using transcriptome-wide principal component analysis (PCA) to compare expression signatures between OSECs and FTSECs, the two cell types largely stratified according to their molecular profiles (Fig. $1 a, b)$. We identified the 87 genes that were the most differentially expressed between OSECs and FTSECs (absolute $\log _{2}$ fold change $[F C]>2, P_{a d j}=10^{-30}$, Fig. 1d, Supplementary Table 2). These included MUC16 (which encodes ovarian cancer screening marker CA125) and CDH1 (E-Cadherin), genes known to be differentially expressed between these cell types (Fig. 1c, Supplementary Fig. 2). Novel, highly overexpressed differentially expressed genes (DEGs) in OSECs compared to FTSECs included GATA4 (FC $\left.=7.1, \mathrm{P}_{\mathrm{adj}}=3.78 \times 10^{-42}\right)$ and NR5A1 $\left(\mathrm{FC}=6.7, \mathrm{P}_{\mathrm{adj}}=2.59 \times 10^{-39}\right)$; both transcriptional activators potentially involved in differentiation of the OSEC phenotype. Novel DEGs highly expressed in FTSECs compared to OSECs include genes that encode the cell surface or secreted proteins $M M P 7\left(F C=9.9, P_{\text {adj }}=1.87 \times 10^{-31}\right)$, CLIC5 $\left(\mathrm{FC}=8.36, \mathrm{P}_{\mathrm{adj}}=5.14 \times 10^{-49}\right)$, TACSTD2 $\left(\mathrm{FC}=8.21, \mathrm{P}_{\mathrm{adj}}=1.2 \times 10^{-42}\right)$ and $C F T R\left(\mathrm{FC}=8.15, \mathrm{P}_{\mathrm{adj}}=\right.$ $\left.7.35 \times 10^{-31}\right)$.

\section{Transcriptomic Relationships between OSECs, FTSECs and HGSOCs}

We applied machine learning algorithms to evaluate the molecular relationships between transcriptomic profiles of OSECs and FTSECs and 394 primary HGSOCs profiled by The Cancer Genome Atlas (TCGA). To correct for differences in read depth and RNA-seq methods between this study and TCGA, we aligned, batch corrected and normalized all three data sets (OSEC, FTSEC and TCGA) together (see Methods). We first defined cell-type specific signatures of OSECs and FTSECs and then applied a One-class logistic regression (OCLR) methodology ${ }^{14}$. Area under the curve (AUC) statistics generated using a leave-one-out approach indicated that the OCLR models performed with high specificity (average AUC for OSECs $=0.99$ and for FTSECs $=0.97$ ). OCLR models provide a score for each sample and for each category, which is rescaled between zero and one, where zero implies no similarity and one implies high similarity. We applied the OCLR models to HGSOCs to evaluate which cell type - OSECs or FTSECs was most highly correlated with HGSOC, thereby indicating which cell type is the most likely cell-of-origin. HGSOC samples were randomized and divided into two equally sized groups $(n=197)$, designated the training set and the validation set. Each set included similar numbers of the four HGSOC molecular subgroups - differentiated, immunoreactive, mesenchymal and proliferative, classified based on gene 
expression signatures. In both the training and validation data sets, we observed a greater proportion of HGSOCs with higher FTSEC scores compared to OSEC scores. In the training set 103/197 tumors (52\%) had an FTSEC score $>0.5$, while only $20 / 197$ tumors $(10 \%)$ had an OSEC score $>0.5$. In the validation set 124/197 tumors (63\%) and 82/197 tumors (42\%) had FTSEC and OSEC scores > 0.5, respectively (Fig. 2a,b). Taken together, these data indicate that the molecular signatures of HGSOCs are more similar to FTSECs than OSECs. There was a weak negative correlation between tumor FTSEC and OSEC scores (Supplementary Fig. 3) (Pearson's product-moment correlation = -0.16, $P=0.002$ ). Consistent with our machine learning observations, in a PCA performed using all expressed genes, FTSECs cluster more closely to HGSOCs than OSECs (Supplementary Fig. 4).

We also evaluated if FTSEC and OSEC scores correlated with clinical and molecular features of HGSOCs ${ }^{15,16}$. In both the training and validation data sets, mesenchymal-type HGSOCs had significantly higher FTSEC OCLR scores $\left(P_{a d j}<0.02\right.$ in the training and validation cohort, Fig. $2 \mathrm{c}, P_{a d j}=8 \times 10^{-4}$ in a meta-analysis of all 394 HGSOCs); this subgroup of HGSOCs exhibit the shortest survival times ${ }^{15}$. By contrast, proliferative type HGSOCs had significantly larger OSEC scores $\left(P_{a d j}<0.001\right.$ in the training and validation cohort, $P_{a d j}=2 \times 10^{-4}$ in a meta-analysis) (Fig. $2 \mathrm{~d}$ ) suggesting that OSECs may be the more likely cell-of origin for this subgroup of HGSOCs. Taken together, these data are consistent with the hypothesis that HGSOCs derive from more than one tissue type ${ }^{17-19}$. Finally, we tested for associations between FTSEC and OSEC OCLR scores and patient age, tumor stage, tumor grade, chemoresponse and debulking status (Supplementary Figs. 5,6). We found no significant associations for FTSECs, but tumors with high OSEC scores were associated with older age at diagnosis $\left(P_{\text {adj }}=0.005\right.$, normalized enrichment score $=$ 1.6). Higher OSEC score was also associated with increased sensitivity to chemotherapy $\left(P_{a d j}=0.03\right.$, normalized enrichment score $=1.5$ ) (Supplementary Fig. 6).

\section{Identifying Epigenomic and Transcriptomic Interactions}

We used chromatin immunoprecipitation sequencing (ChIP-seq) to characterize epigenomic landscapes in OSECs, FTSECs and HGSOCs, focusing on super-enhancers (SEs), which are dense clusters of highly active chromatin that typically localize with master regulators of cellular identity. We generated ChIP-seq data for acetylated H3K27ac in five primary HGSOC tissues; H3K27ac ChIP-seq data for normal OSEC and FTSEC cells have been reported previously ${ }^{20}$. H3K27ac profiles were used to map SEs in each tissue type. OSECs and HGSOCs had the largest numbers of cell-type specific SEs ( $n=337$ and $n=336$, respectively). FTSECs had the lowest number of unique SEs $(n=173)$; this was due largely to the fact that there were significantly more SEs shared between FTSECs and HGSOCs $(n=80)$ than between OSECS and HGSOCs $(n=37)$ (odds ratio $=12.9$, Fishers Exact Test, $p<2.2 \times 10^{-16}$, Fig. 3a,b). Using the 
transcriptomic data described above, we verified tissue-specific overexpression of genes located at tissuerelevant SE loci (Fig. 3c,f,i), and identified novel candidate target genes regulated by SEs in each cell type . These included SULT1B1 in OSECs (Fig. 3d,e); the Tripartite Motif Containing 55 (TRIM55) gene in FTSECs (Fig. 3g,h) and the SOX18 transcription factor in HGSOCs (Fig. 3j,k). Of particular note, the PAX8 transcription factor was overexpressed in both FTSECs and HGSOCs and coincides with a SE detected in both cell types at this locus; PAX8 is a well-established biomarker that is ubiquitously expressed in FTSECs and is overexpressed in the majority of primary HGSOCs ${ }^{21-23}$ (Supplementary Fig. 7).

Finally, we performed a targeted analysis of genes associated with DNA binding, transcription factor activity and chromatin remodeling to identify putative novel drivers of transcriptional deregulation during HGSOC development from FTSECs and/or OSECs. For nine of the most overexpressed transcriptional regulators in HGSOCs (Fig. 4a), high-quality ChIP-seq data were available from cistrome.org. Using these ChIP-seq data sets we quantified how many of the most differentially expressed genes in HGSOCs were located near to (within $50 \mathrm{kbp}$ ) a factor-specific peak compared to matched random peaks (see Methods). Factor-specific peaks for regulators including SPI1, CTCFL, NFE2, ASCL2 and GRHL2 were more numerous in the vicinity of HGSOC DEGs $\left(P<10^{-30}\right.$ for a comparison to FTSECs and $P<10^{-50}$ for a comparison to OSECs) than randomly generated matched sets of background peaks (100 iterations, $P<$ 0.01 Fig. 4b,c; genes located close to factor peaks are listed in Supplementary Tables 3,4). Binding sites for ELF3, a factor highly expressed in both FTSECs and HGSOCs but lowly expressed in OSECs were specifically enriched near to genes differentially expressed between FTSECs and HGSOCs $(P=0.009$, Fig. $4 \mathrm{~b})$, with no evidence of enrichment in the set of genes differentially expressed in the development of HGSOC from OSECs ( $P=1$, Fig. 4c). Conversely EHF binding sites were associated with DEGs in a comparison of HGSOCs to OSECs but not FTSECs $(P=0.009$ and $P=0.06$, respectively). Notably, both ELF3 and EHF reside at SEs in HGSOC (Supplementary Figs. 8,9). Collectively these factors represent novel drivers of transcriptional reprogramming in HGSOC, with ELF3 likely to be specific to the transformation of FTSECs, and EHF a putative driver of HGSOC development from OSECs.

\section{Discussion}

There remains debate about the cellular origins of high-grade serous ovarian cancer. Over the last few years the fallopian tube, and specifically the secretory epithelial cell component (FTSECs), has emerged as a likely origin. This is based on the identification of early stage precursor lesions in the fallopian tube that express secretory cell lineage markers and harbor TP53 mutations, particularly in fallopian tube fimbriae ${ }^{3,4,8,10,24}$. In addition, in vitro and in vivo models studies ${ }^{12,25,26}$ support FTSECs as a major cell-of- 
origin for HGSOC and salpingectomy (surgical removal of fallopian tubes but not the ovaries) can reduce the risk of ovarian cancer by around $35 \%$ or more ${ }^{27}$. However, the current data do not implicate FTSECS as precursors of every HGSOC, in particular in non-familial cases (i.e. non-carriers of $B R C A 1 / B R C A 2$ mutations). This suggests that alternative cellular precursors may exist, which supported the present reevaluation of ovarian surface epithelial cells (OSECs), historically thought to the precursor of HGSOCs. Collectively, in a cohort of almost 400 HGSOCs, we found greater similarities in transcriptomic signatures between FTSECs and HGSOCs than between OSECs and HGSOCs. Primary HGSOCs can be substratified into 4 different molecular groups based on mRNA expression profiles and high FTSEC scores were enriched in mesenchymal-type HGSOCs, consistent with previous observations indicating that fallopian-like HGSOCs are associated with poorer outcomes ${ }^{28}$. OSEC profiles were most closely related to the proliferative-type HGSOCs associated with better outcomes. These data imply that HGSOC molecular subgroups may have different precursors, with OSECs the putative cell-of-origin for the proliferative subgroup. While our results add to a growing body of evidence supporting FTSECs as the predominant cellof-origin for HGSOC ${ }^{9,19,29}$, there remains a substantial and often overlooked body of evidence that supports OSECs as alternative precursors in a subgroup of cases. OSECs can express many HGSOC markers, including PAX8 ${ }^{2,30}$. OSECs from primary ovaries and OSEC cultures from women at high-risk of ovarian cancer show phenotypic differences compared to OSEC cultures from non-inherited cases, with OSECS from high-risk women more committed to an epithelial phenotype and maintaining expression of CA125 longer than OSECs from non-high-risk women ${ }^{31}$. In addition, occult cancers have been detected in the ovaries of women undergoing prophylactic risk reducing oophorectomy and can occur without evidence of lesions in the fallopian tube ${ }^{32}$. Our results are also consistent with a recent detailed analysis of almost 60 ovaries which documented evidence for metaplasia of ovarian epithelium to a Müllerian phenotype, and proposed that this could be an early step in the development of serous tumors ${ }^{2}$.

While our study shows clear differences between the transcriptomic profiles of OSECs and FTSECs, there are some caveats to the study design that could influence interpretation of our findings. One of these is the potential impact of short term culture on the gene expression profiles of primary OSECs and FTSECs. Neither cell type is readily amenable to microdissection from primary tissues - OSECs are a scarce population on the surface of the ovary and FTSECs are intercalated with ciliated cells not currently thought to be precursors of serous carcinogenesis; thus culturing cells was our preferred approach to obtaining sufficient purified OSEC/FTSEC cell material on which to perform in-depth molecular profiling studies without having to pool samples across patients. To limit artifacts induced by cell culture, cells were not genetically modified and the procedures for primary culture were optimized for each cell type specifically. 
Moreover, our results are closely reflected by results of a recent molecular profiling study which compared an independent cohort of HGSOCs to signatures derived from a small cohort of fallopian ( $\mathrm{n}=3$ pooled samples) or ovarian brushing ( $n=4$ pooled samples) and observed similar trends: that the majority of HGSOCs correlate with fallopian signatures but a subset of cases show higher correlation with OSEC signatures ${ }^{29}$; suggesting that short-term culture did not substantially influence our analyses.

Little is known about the key transcription factors (TFs) driving oncogenesis in HGSOC. PAX8 is a TF known to be highly expressed in FTSECs and functionally involved in disease development ${ }^{22,30,33-35}$. We found that PAX8 is marked by a super-enhancer in both FTSECs and HGSOCs. We also identified ELF3 and EHF as putative drivers of the transformation of FTSECs and OSECs respectively, both of which have been implicated in ovarian cancer development ${ }^{36,37}$. Of the four genes highly expressed in OSECs, two were transcriptional regulators: GATA4 is a developmental zinc finger TF and NR5A1 is a transcriptional activator involved in sex determination and differentiation of steroidogenic tissues. Both GATA4 and NR5A1 were highly expressed in proliferative type HGSOCs, suggesting these TF networks may specifically be involved in the development of this tumor subgroup.

Compared to previous studies, these investigations represent a significant advance in both scale and scope of the molecular profiling performed ${ }^{28}$. While this study focused on the HGSOC subtype of ovarian cancer, where there is good evidence for the likely precursor cell types, a similar approach could also be used to investigate the cells of origin of other histotypes; OSECs, FTSECs and other Müllerian epithelia should also be comprehensively evaluated as putative cells-of-origin for low-grade serous, mucinous, clear cell and endometrioid ovarian cancers. In summary, this study provides novel insights into the etiology and biology of likely precursors of HGSOC, ovarian surface and fallopian tube secretory epithelial cells. In addition we have described for the first time, the putative transcriptional regulators that are associated with these cell types and their role in HGSOC development; these represent candidate clinical biomarkers and novel therapeutic targets for this disease. 


\section{Methods}

\section{Patient consent and IRB approval}

All specimens were collected from University College Hospital (London, UK), LAC + USC Medical Center (Los Angeles, CA, USA) and Oregon Health \& Science University (Portland, OR, USA). All were collected with informed patient consent and Institutional Review Board approval.

\section{Sample collection, RNA extraction and RNA sequencing}

OSECs and FTSECs were harvested from women diagnosed with ovarian, uterine or cervical cancer, and were histologically normal. Short-term cultures were established as previously described ${ }^{12,13}$. Briefly, OSECs were harvested using a cytobrush and cultured in NOSE-CM media containing 15\% fetal bovine serum (FBS, Hyclone), $34 \mathrm{\mu g} \mathrm{ml}^{-1}$ bovine pituitary extract, $10 \mathrm{ng} \mathrm{ml}^{-1}$ epidermal growth factor (Life Technologies), $5 \mathrm{\mu g} \mathrm{ml}^{-1}$ insulin and $500 \mathrm{ng} \mathrm{ml}^{-1}$ hydrocortisone (Sigma-Aldrich). FTSECs were harvested by Pronase/DNase I digestion (Roche and Sigma-Aldrich, respectively) for $48-72$ hours at $4^{\circ} \mathrm{C}$ and cultured on collagen I (Sigma-Aldrich) using DMEM/F12 base media supplemented with 2\% Ultroser G (Pall Corporation). At $\sim 80 \%$ confluency, cells were lysed using the QIAzol reagent and RNA extracted using the RNeasy Mini kit (both QIAgen). RNA sequencing was performed by the University of Southern California Epigenome Core Facility.

\section{RNAseq data processing and QC}

All data analysis was performed using ' $R$ ' and 'Bioconductor', and packages therein. RNAseq data for 394 HGSOC samples was obtained from The Cancer Genome Atlas (TCGA) data portal as protected data (raw sequencing, fastq files) and downloaded via CGHub's geneTorrent. Data was aligned to a reference genome (hg19) using STAR. And quality control of aligned samples performed using RSeQC. GC bias and batch effect corrections were performed using EDASeq and 'sva'. To adjust for batch effects we used an empirical Bayes framework (comBat), available in 'sva'.

\section{Differential gene expression analyses}

After normalization, the data matrix contained 21,071 genes. Parametric statistics (Student's T-test) and supervised hierarchical clustering were performed to identify genes differentially expressed in pairwise comparisons of two groups of interest (OSEC, FTSEC and HGSOC). P-values were adjusted using Benjamini-Hochberg step-up procedure.

\section{Machine learning analyses}

We applied a machine learning approach to define a probabilistic score associated to both normal cell types, and infer tumor origins. A One-class classifier was selected as this method can handle non-traditional 
supervised scenarios where no negative class can be defined. The classifiers were implemented by the gelnet R-package version 1.2.1. Data were mean centered considering all samples together, then each cell type used separately to train and test the models. To train the OSEC model we considered all OSEC samples, with a coefficient for the L1-norm penalty equal to 0 and coefficient for the L2-norm penalty equal to 1 as arguments of gelnet function. We then evaluated the model performance through leave-one-out procedure where the left-out OSEC sample was mixed into FTSEC sample background. The accuracy was evaluated via the Area Under the ROC curve method, with $99 \%$ of OSEC samples correctly predicted, on average, and $97 \%$ of FTSECs correctly predicted. We then tested the model prediction behaviour when applied to HGSOCs from TCGA. We took advantage of the fast gene set enrichment analysis (fgsea, version 1.2.1, http://bioconductor.org/packages/fgsea/) method to evaluate enrichment of clinical attributes across the tumor OCLR scores from both FTSEC and OSEC models. We applied the fgsea function with the parameter nperm equal to 10,000 .

\section{Tissue ChIP-seq in HGSOC specimens}

Tissue ChIP-seq was performed based on the methods described in Pomerantz et al $2015 .^{38}$. One $3 \mathrm{~mm}$ core was isolated from an epithelial-rich portion of tumor, and pulverized using the Covaris CryoPrep system (Covaris, Woburn, MA), set to an intensity of 4 . Tissues were fixed using $1 \%$ formaldehyde (Thermo fisher, Waltham, MA) for 10 minutes at room temperature. Fixation was quenched with $125 \mathrm{mM}$ glycine and samples were rinsed with cold PBS before a 10 minute lysis in a buffer containing $50 \mathrm{mM}$ Tris, $10 \mathrm{mM}$ EDTA, $1 \%$ SDS with protease inhibitor). Chromatin was sheared to 300-500 base pairs and 5 volumes dilution buffer (1\% Triton X-100, 2 mM EDTA, $150 \mathrm{mM} \mathrm{NaCl}, 20 \mathrm{mM}$ Tris $\mathrm{HCl}$ pH 8.1) added. Each sample was incubated with 1 mg H3K27ac antibody (DiAGenode, C15410196, Denville, NJ) coupled with protein A and protein $\mathrm{G}$ beads (Life Technologies, Carlsbad, CA) at $4^{\circ} \mathrm{C}$ overnight. Immunoprecipitated chromatin was washed with RIPA buffer (0.05M HEPES pH 7.6, 1 mM EDTA, 0.7\% Na Deoxycholate, 1\% NP-40, $0.5 \mathrm{M} \mathrm{LiCl})$ five times and rinsed with TE buffer $(\mathrm{pH} 8.0)$ once. The sample was resuspended in elution buffer (50mM Tris, $10 \mathrm{mM}$ EDTA, $1 \%$ SDS), treated with RNase for 30 minutes at $37^{\circ} \mathrm{C}$, and incubated with proteinase $\mathrm{K}$ overnight at $65^{\circ} \mathrm{C}$. Sample DNA and $1 \%$ input were extracted, and sequencing libraries prepared using the ThruPLEX-FD Prep Kit (Rubicon Genomics, Ann Arbor, MI). Libraries were sequenced using 75-base pair single reads on the Illumina platform (Illumina, San Diego, CA) at the Dana-Farber Cancer Institute.

\section{ChIP-seq data analysis}

The AQUAS pipeline (https://github.com/kundajelab/chipseq pipeline) was used to processed ChIP-seq data. Reads were aligned to the reference human genome (hg19), filtered by read quality and duplicate 
reads removed. macs2 (https://pypi.python.org/pypi/MACS2) was used for peak calling. For the cell lines, two technical replicates were generated and the final peaks were obtained using a naive overlap approach, where the peaks are included if they overlap more than $50 \%$ between the two technical replicates. We have previously described H3K27ac ChIP-seq for immortalized OSEC and FTSEC lines ${ }^{20}$. Immortalized OSECs have been previously show to be representative of unmodified cells ${ }^{39}$. We verified that the expression profiled of immortalized FTSECs used in this study clustered with primary FTSECs (Supplementary Figure 10). After alignment, homer (http://homer.ucsd.edu/homer/) was used to identify super-enhancers, using a super slope parameter of 2 and a minimum distance of ten thousand. For defining a set of HGSOC SEs, we selected SEs that were called in at least two HGSOC samples. For the FTSEC set of SEs, SEs were called individually in each technical replicate, then, all the SEs that overlapped both technical replicates within the same cell line (FTSEC33 or FTSEC246) were selected to get the union set. We used a similar approach to get the union set of SEs for the OSEC cell lines.

\section{Cistrome TF analyses}

From the Cistrome database (cistrome.org), we selected and downloaded BED peak files for experiments passing 3/6 quality control filters. Data sets used are listed in the Online Methods. The following data sets were included: HOXC9: GSM848788, GSM848789; LXH2: GSM1208644,GSM1567049; GLIS1:

ENCSR482BBZ, GSM1208752, GSM2026832, GSM803384; GATA4: ENCSR590CNM, GSM1505646, GSM1505647, GSM1505651, GSM1505657; HNF1B: GSM1239408, GSM1505680, GSM1505681, GSM1505682, GSM1505683; EHF: GSM1208609, GSM1548071, GSM1548072; OVOL2: GSM1239518; ELF3: GSM1208732; SPI1: GSM1010843, GSM1370280, GSM1370292, GSM1421017, GSM1642769, GSM1703900; CTCFL: GSM1239559, GSM1817655, GSM1817659, GSM1817663, GSM1817668, GSM1817669, GSM803401; PITX1: GSM1208667; NFE2: GSM1067276, GSM1427076, GSM935414, GSM935652; ASCL2: GSM1208591; GRHL2: GSM1125982, GSM1125983, GSM1125984, GSM1239569. We converted hg18 genomic positions to hg19 coordinates using the liftOver tool from UCSC (http://genome.ucsc.edu/cgi-bin/hgLiftOver). Where multiple data sets existed for a given factor, data set were merged to create a union peak set. Random peaks were generated maintaining the same chromosome proportion in the original peak file to create 100 iterations in each random peak simulation. To identify the number of genes associated to cistrome peaks we considered a distance window of $50 \mathrm{kbp}$ from each TF peak coordinates using the distance() function from GenomicRanges package version 1.28.4. The P-value was calculated using the following expression: $P=(r+1) /(n+1)$, where $r$ is the number of random values greater or equal than observation value and $\mathrm{n}$ is the number of random iterations.

\section{Data and code availability}


All raw RNA sequencing data are also accessible at http://tcgabiolinks.fmrp.usp.br/OvarianRNAseq/. The code used to perform the analyses is available as an Rmarkdown at:

http://tcgabiolinks.fmrp.usp.br/OvarianRNAseq/.

\section{Acknowledgements}

The results shown here are in part based upon data generated by the TCGA Research Network:

http://cancergenome.nih.gov/. Some of the normal tissue specimens were collected as part of the USC Jean

Richardson Gynecologic Tissue and Fluid Repository, which is supported by a grant from the USC

Department of Obstetrics \& Gynecology and the NCT Cancer Center Shared Grant award P30 CA014089

(to the Norris Comprehensive Cancer Center). K.L. is supported in part by a K99/R00 Pathway to Independence Award from the NIH (R00CA184415), institutional support from the Samuel Oschin Comprehensive Cancer Institute at Cedars-Sinai Medical Center and a Career Development Award from The Tower Cancer Research Foundation. H.N. and M.A.S.F. are supported by grants 2015/07925-5 and 2017/08211-1 from Sao Paulo Research Foundation (FAPESP). H.N. is also supported by an institutional grant (Henry Ford Hospital). This work was supported in part by the Ovarian Cancer Research Fund Alliance Program Project Development Grant (373356): Co-Evolution of Epithelial Ovarian Cancer and Tumor Stroma. Additional support for this work came from NIH/NCI grants 1R01CA211707 and 1R01CA207456 and OCRF award 258807. 


\section{Figure Legends}

Figure 1. Transcriptomic profiling of OSECs and FTSECs. (a \& b) Principal component analysis (PCA) of RNA-seq profiles of OSECs and FTSECs. OSEC samples tend to cluster more tightly together whereas FTSEC samples show more diffuse clustering. This suggests greater inter-patient heterogeneity between for FTSEC samples. (c) Volcano plot illustrating differential gene expression between OSEC and FTSEC samples. Known cell-type specific markers for each cell type are indicated.

Figure 2. One-class logistic regression (OCLR) predictors of the cellular origins of HGSOC. (a \& b) OSEC and FTSEC whole transcriptomic signatures were developed and compared to whole transcriptomic signatures of 394 primary HGSOCs publicly available from TCGA project. TCGA analyses were divided into (a) test $(n=197)$ and (b) validation $(n=197)$ sets of tumors. In both test and validation sets, FTSEC score tended to be higher in HGSOCs than OSEC scores. The dashed line indicates a score of 0.5. (c \& d) FTSEC and OSEC signatures were compared across HGSOC molecular subgroups. FTSEC score was highest in the mesenchymal subgroup in both test and validation sets; (c) OSECs were highest in the proliferative subgroup.

Figure 3. Super-enhancer-gene relationships in OSECs, FTSECs and HGSOCs. (a) Pseudo Venn diagram of the SE catalogue of OSECS, FTSECs and HGSOCs, showing SE intersections across the three tissue types. Although fewer SEs were catalogued in FTSECs compared to OSECs and HGSOCs, significantly more SEs are shared between FTSECs and HGSOCs than between OSECs and HGSOCs (Fishers Exact Test, $\mathrm{p}<2.2 \times 10^{-16}$ ). (b) The landscape of cell-type specific SEs across OSECs, FTSECs and HGSOCs. For OSECs and FTSECs, H3K27ac ChIP-seq data generated for two independent immortalized normal lines per cell type were used to identify SEs. For HGSOCs, H3K27ac ChIP-seq data were generated for five different primary HGSOCs. (c-k) Tissue-specific SEs associated with elevated gene expression in cis in a cell-type specific manner. (c-e), OSEC-specific SEs, (f-h) FTSEC-specific SEs, (i-k) HGSOC-specific SEs. (c,f,i) H3K27ac ChIPseq data were integrated with RNA-seq data for each tissue type. Average gene expression in 114 OSECs, 74 FTSECs and 394 HGSOCs is shown for regions centered on cell-type specific SEs; (d,g,j) Representative loci displaying tissue-specific SE deposition for each tissue type; $(e, h, k)$ Box plots illustrating differential gene expression between tissue types for candidate, cell type specific cis-regulated genes. The associated gene consistently displays higher expression in the SE-positive tissue type.

Figure 4. Novel transcriptional regulators implicated in HGSOC development. (a) Transcriptional regulators highly expressed in HGSOC. The number of differentially expressed genes in HGSOCs 
bioRxiv preprint doi: https://doi.org/10.1101/330597; this version posted May 25, 2018. The copyright holder for this preprint (which was not certified by peer review) is the author/funder. All rights reserved. No reuse allowed without permission.

compared to (b) FTSECs and (c) OSECs that localize with ChIP-seq peaks for each factor (orange) and factor-specific matched random peaks (blue). ${ }^{*} P>0.05,{ }^{* *} P>0.01$. 


\section{References}

1. Kruk, P. A. \& Auersperg, N. Human ovarian surface epithelial cells are capable of physically restructuring extracellular matrix. Am. J. Obstet. Gynecol. 167, 1437-1443 (1992).

2. Park, K. J. et al. Observations on the Origin of Ovarian Cortical Inclusion Cysts in Women Undergoing Risk-Reducing Salpingo-Oophorectomy. Histopathology (2017). doi:10.1111/his.13444

3. Paley, P. J. et al. Occult cancer of the fallopian tube in BRCA-1 germline mutation carriers at prophylactic oophorectomy: a case for recommending hysterectomy at surgical prophylaxis. Gynecol. Oncol. 80, 176-180 (2001).

4. Callahan, M. J. et al. Primary fallopian tube malignancies in BRCA-positive women undergoing surgery for ovarian cancer risk reduction. J. Clin. Oncol. 25, 3985-3990 (2007).

5. Piek, J. M. et al. Dysplastic changes in prophylactically removed Fallopian tubes of women predisposed to developing ovarian cancer. J. Pathol. 195, 451-456 (2001).

6. Leeper, K. et al. Pathologic findings in prophylactic oophorectomy specimens in high-risk women. Gynecol. Oncol. 87, 52-56 (2002).

7. Medeiros, F. et al. The tubal fimbria is a preferred site for early adenocarcinoma in women with familial ovarian cancer syndrome. Am. J. Surg. Pathol. 30, 230-236 (2006).

8. Gilks, C. B. et al. Incidental nonuterine high-grade serous carcinomas arise in the fallopian tube in most cases: further evidence for the tubal origin of high-grade serous carcinomas. Am. J. Surg. Pathol. 39, 357-364 (2015).

9. Labidi-Galy, S. I. et al. High grade serous ovarian carcinomas originate in the fallopian tube. Nat. Commun. 8, 1093 (2017).

10. Kindelberger, D. W. et al. Intraepithelial carcinoma of the fimbria and pelvic serous carcinoma: Evidence for a causal relationship. Am. J. Surg. Pathol. 31, 161-169 (2007).

11. Staub, E., Buhr, H. J. \& Gröne, J. Predicting the site of origin of tumors by a gene expression signature derived from normal tissues. Oncogene 29, 4485-4492 (2010).

12. Karst, A. M., Levanon, K. \& Drapkin, R. Modeling high-grade serous ovarian carcinogenesis from the fallopian tube. Proc Natl Acad Sci USA 108, 7547-7552 (2011).

13. Lawrenson, K. et al. In vitro three-dimensional modelling of human ovarian surface epithelial cells. Cell Prolif. 42, 385-393 (2009).

14. Sokolov, A., Paull, E. O. \& Stuart, J. M. One-class detection of cell states in tumor subtypes. Pac. Symp. Biocomput. 21, 405-416 (2016).

15. Cancer Genome Atlas Research Network. Integrated genomic analyses of ovarian carcinoma. Nature 474, 609-615 (2011).

16. Tothill, R. W. et al. Novel molecular subtypes of serous and endometrioid ovarian cancer linked to clinical outcome. Clin. Cancer Res. 14, 5198-5208 (2008).

17. Pothuri, B. et al. Genetic analysis of the early natural history of epithelial ovarian carcinoma. PLoS ONE 5, e10358 (2010).

18. Salazar, H. et al. Microscopic benign and invasive malignant neoplasms and a cancer-prone phenotype in prophylactic oophorectomies. J Natl Cancer Inst 88, 1810-1820 (1996).

19. Eckert, M. A. et al. Genomics of ovarian cancer progression reveals diverse metastatic trajectories including intraepithelial metastasis to the fallopian tube. Cancer Discov. 6, 1342-1351 (2016).

20. Coetzee, S. G. et al. Cell-type-specific enrichment of risk-associated regulatory elements at ovarian cancer susceptibility loci. Hum. Mol. Genet. 24, 3595-3607 (2015).

21. Mhawech-Fauceglia, P. et al. Pair Box 8 (PAX8) protein expression in high grade, late stage (stages III and IV) ovarian serous carcinoma. Gynecol. Oncol. 127, 198-201 (2012).

22. Cheung, H.W. et al. Systematic investigation of genetic vulnerabilities across cancer cell lines reveals lineage-specific dependencies in ovarian cancer. Proc Natl Acad Sci USA 108, 12372-12377 (2011). 
23. Laury, A. R. et al. A comprehensive analysis of PAX8 expression in human epithelial tumors. Am. J. Surg. Pathol. 35, 816-826 (2011).

24. Kuhn, E. et al. TP53 mutations in serous tubal intraepithelial carcinoma and concurrent pelvic highgrade serous carcinoma--evidence supporting the clonal relationship of the two lesions. J. Pathol. 226, 421-426 (2012).

25. Perets, R. et al. Transformation of the fallopian tube secretory epithelium leads to high-grade serous ovarian cancer in Brca;Tp53;Pten models. Cancer Cell 24, 751-765 (2013).

26. Zhai, Y. et al. High-grade serous carcinomas arise in the mouse oviduct via defects linked to the human disease. J. Pathol. 243, 16-25 (2017).

27. Falconer, H., Yin, L., Grönberg, H. \& Altman, D. Ovarian cancer risk after salpingectomy: a nationwide population-based study. J Natl Cancer Inst 107, (2015).

28. Merritt, M. A. et al. Gene expression signature of normal cell-of-origin predicts ovarian tumor outcomes. PLOS ONE 8, e80314 (2013).

29. Ducie, J. et al. Molecular analysis of high-grade serous ovarian carcinoma with and without associated serous tubal intra-epithelial carcinoma. Nat. Commun. 8, 990 (2017).

30. Adler, E., Mhawech-Fauceglia, P., Gayther, S. A. \& Lawrenson, K. PAX8 expression in ovarian surface epithelial cells. Hum. Pathol. 46, 948-956 (2015).

31. Dyck, H. G. et al. Autonomy of the epithelial phenotype in human ovarian surface epithelium: changes with neoplastic progression and with a family history of ovarian cancer. Int. J. Cancer 69, 429-436 (1996).

32. Powell, C. B. et al. Risk-reducing salpingo-oophorectomy in BRCA mutation carriers: role of serial sectioning in the detection of occult malignancy. J. Clin. Oncol. 23, 127-132 (2005).

33. Elias, K. M. et al. Epigenetic remodeling regulates transcriptional changes between ovarian cancer and benign precursors. JCI Insight 1, (2016).

34. Kar, S. P. et al. Enrichment of putative PAX8 target genes at serous epithelial ovarian cancer susceptibility loci. Br. J. Cancer 116, 524-535 (2017).

35. Adler, E. K. et al. The PAX8 cistrome in epithelial ovarian cancer. Oncotarget 8, 108316-108332 (2017).

36. Brenne, K., Nymoen, D. A., Hetland, T. E., Trope', C. G. \& Davidson, B. Expression of the Ets transcription factor EHF in serous ovarian carcinoma effusions is a marker of poor survival. Hum. Pathol. 43, 496-505 (2012).

37. Yeung, T.-L. et al. ELF3 is a negative regulator of epithelial-mesenchymal transition in ovarian cancer cells. Oncotarget 8, 16951-16963 (2017).

38. Pomerantz, M. M. et al. The androgen receptor cistrome is extensively reprogrammed in human prostate tumorigenesis. Nat. Genet. 47, 1346-1351 (2015).

39. Li, N. F. et al. Human ovarian surface epithelial cells immortalized with hTERT maintain functional pRb and p53 expression. Cell Prolif. 40, 780-794 (2007). 
bioRxiv preprint doi: https://doi.org/10.1101/330597; this version posted May 25, 2018. The copyright holder for this preprint (which was not Figure 1 certified by peer review) is the author/funder. All rights reserved. No reuse allowed without permission.

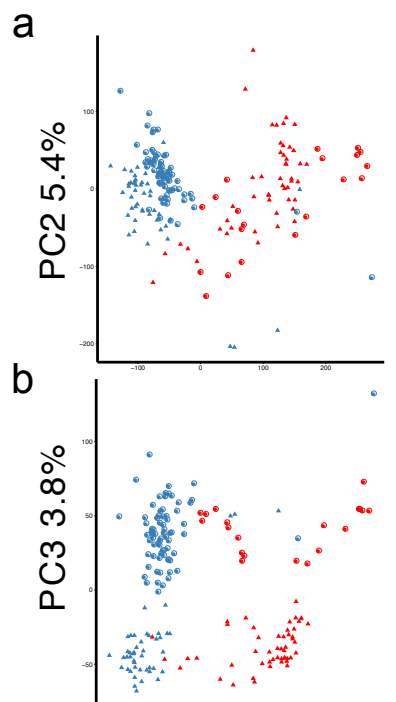

PC1 21\%

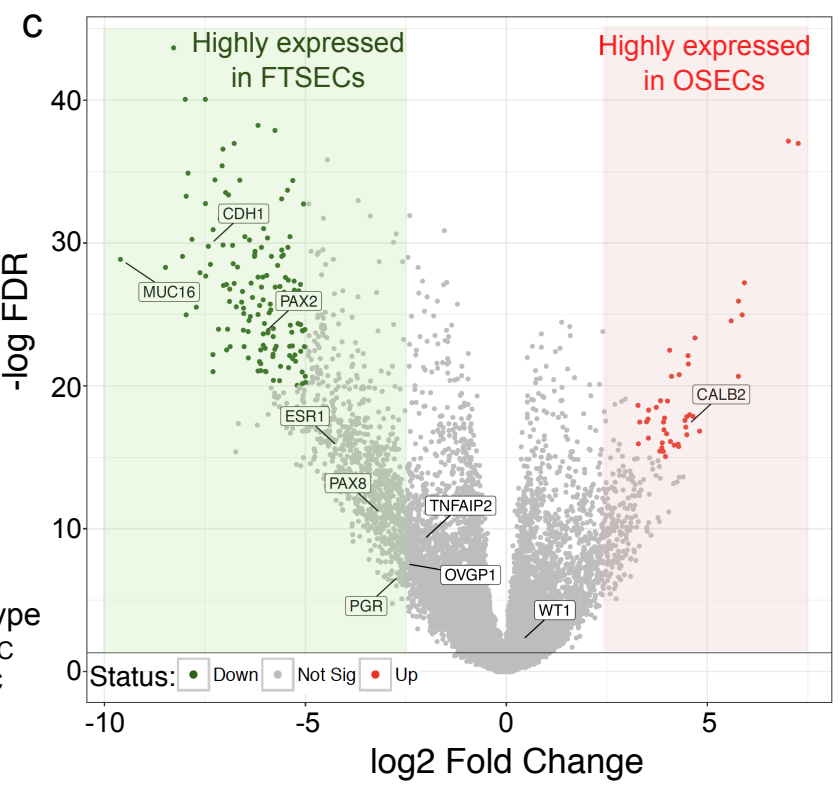


bioRxiv preprint doi: https://doi.org/10.1101/330597; this version posted May 25, 2018. The copyright holder for this preprint (which was not

Figure 2

a

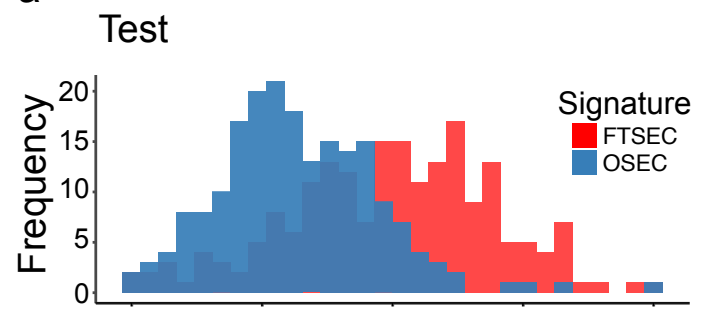

b

b Validation

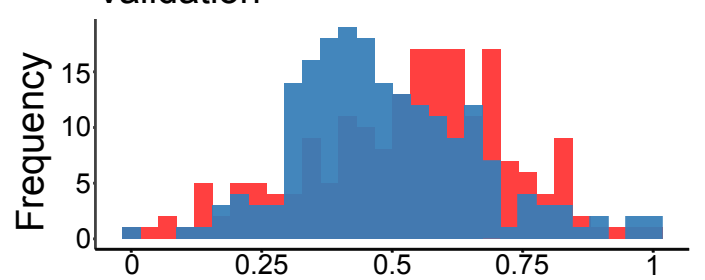

OSEC/FTSEC Score
C

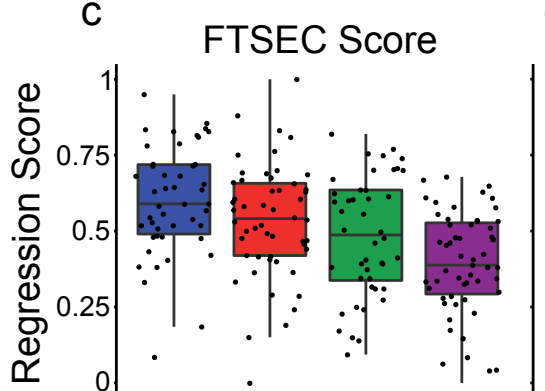

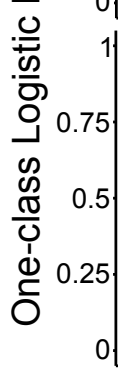
. OSEC Score

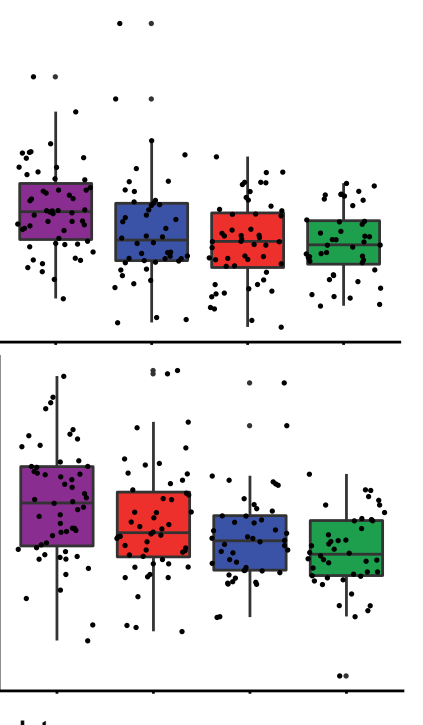
Subtypes

† Mesenchymal Differentiated Immunoreactive Proliferative 
bioRxiv preprint doi: https://doi.org/10.1101/330597; this version posted May 25, 2018. The copyright holder for this preprint (which was not

Figure 2

a

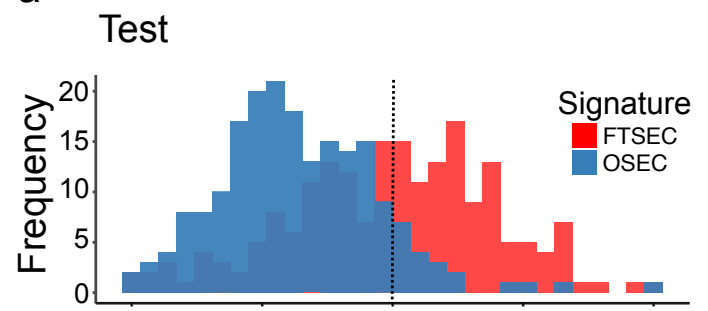

b Validation

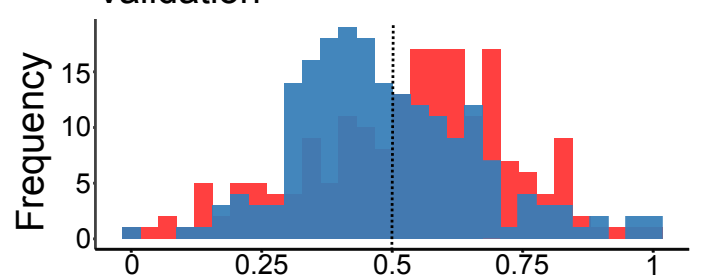

OSEC/FTSEC Score
C

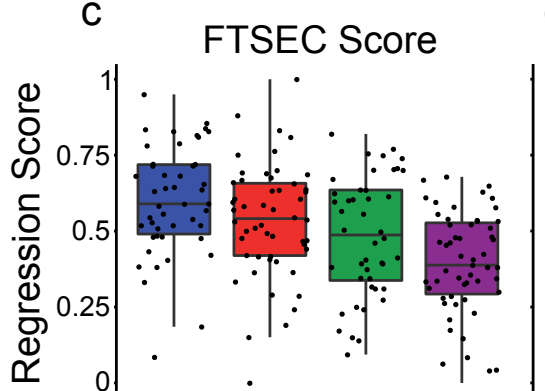

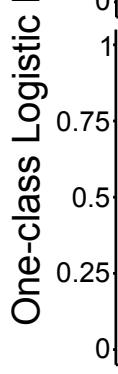
. OSEC Score

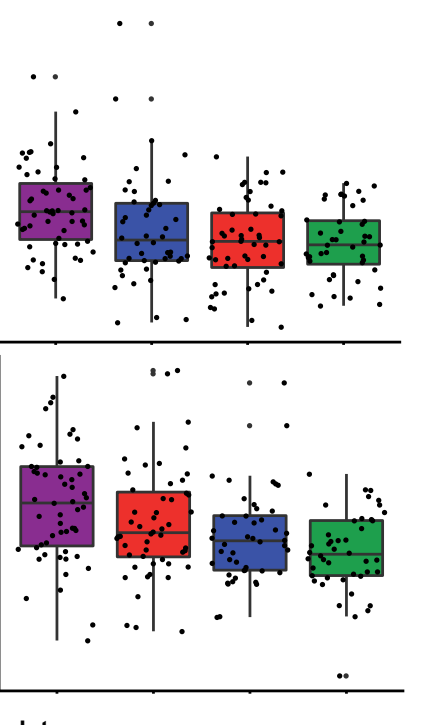
Subtypes

† Mesenchymal Differentiated Immunoreactive Proliferative 
Figure 3

bioRxiv preprint doi: https://doi.org/10.1101/330597; this version posted May 25, 2018. The copyright holder for this preprint (which was not certified by peer review) is the author/funder. All rights reserved. No reuse allowed without permission.

a

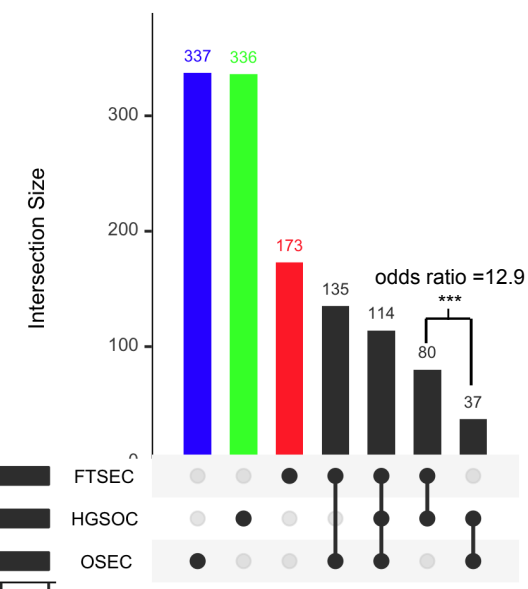

b

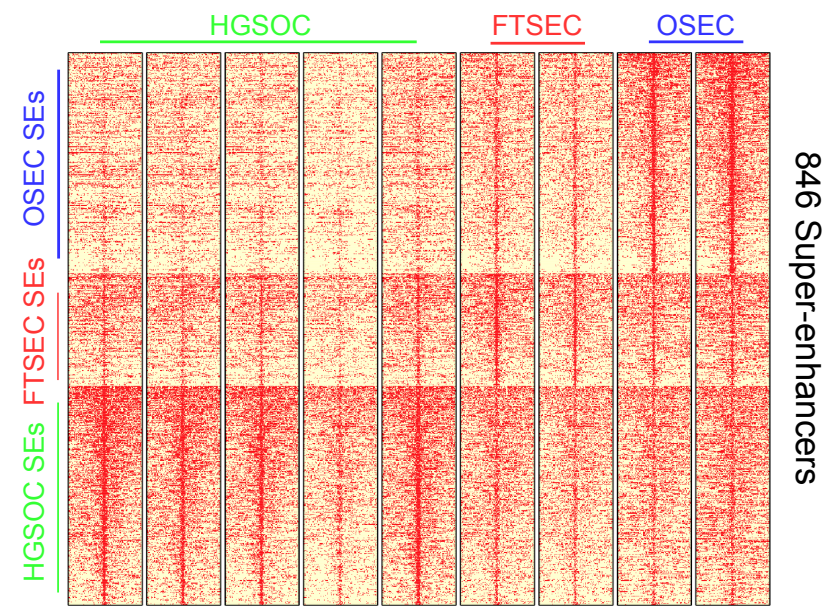

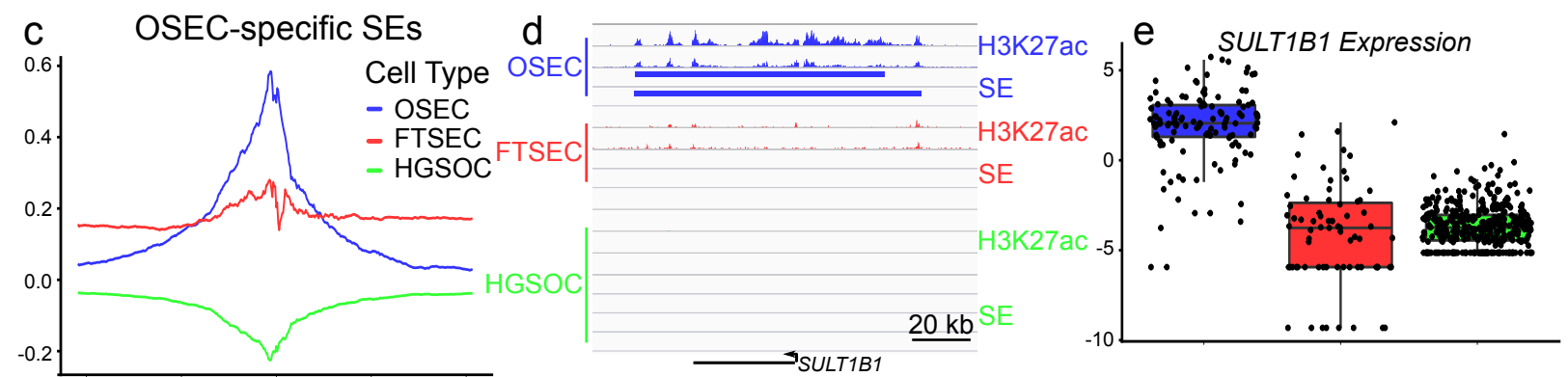

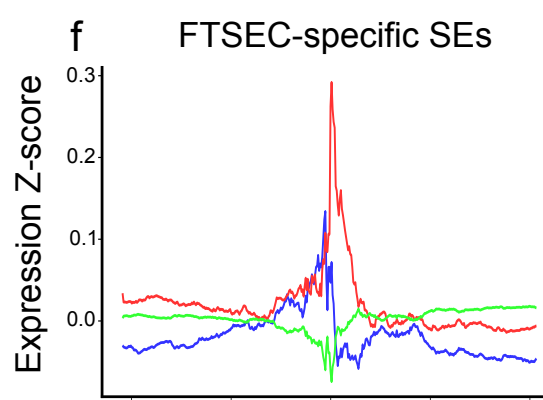

OSEC

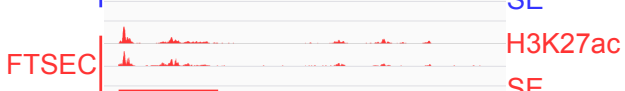

H3K27ac $\mathrm{h}$ TRIM.55 Expression
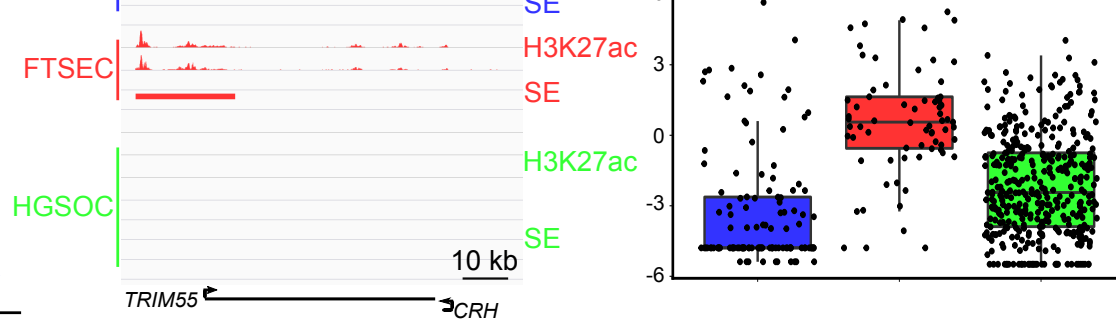

i HGSOC-specific SES
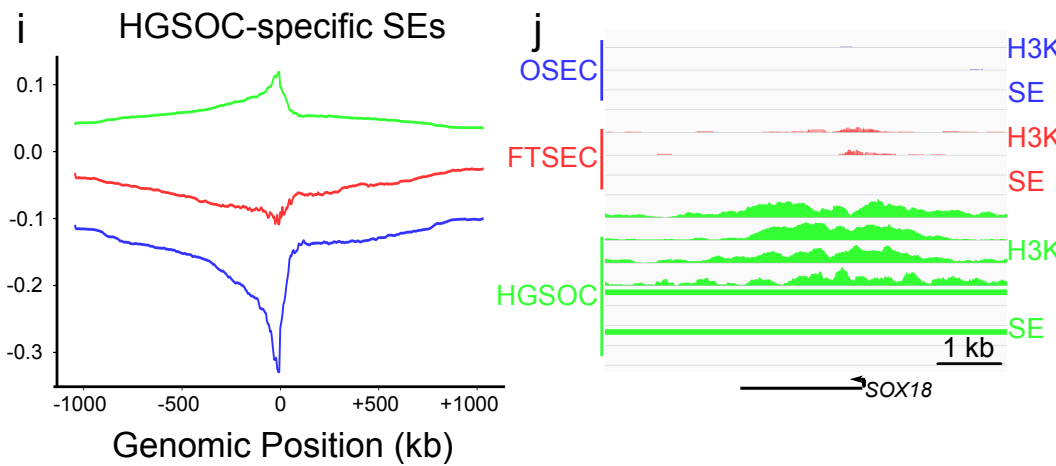

H3K27ac

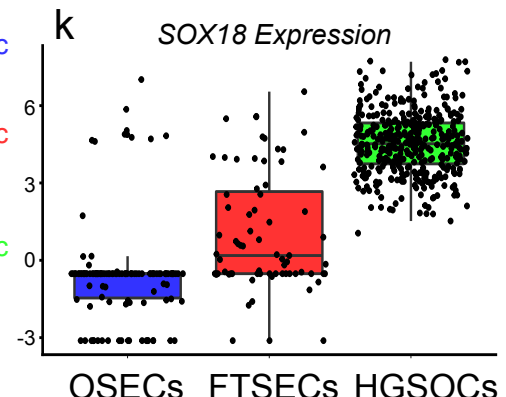

OSECs FTSECs HGSOCs

Cell Type 
Figure 4

bioRxiv preprint doi: https://doi.org/10.1101/330597; this version posted May 25, 2018. The copyright holder for this preprint (which was not certified by peer review) is the author/funder. All rights reserved. No reuse allowed without permission.

a

Factor Gene Expression

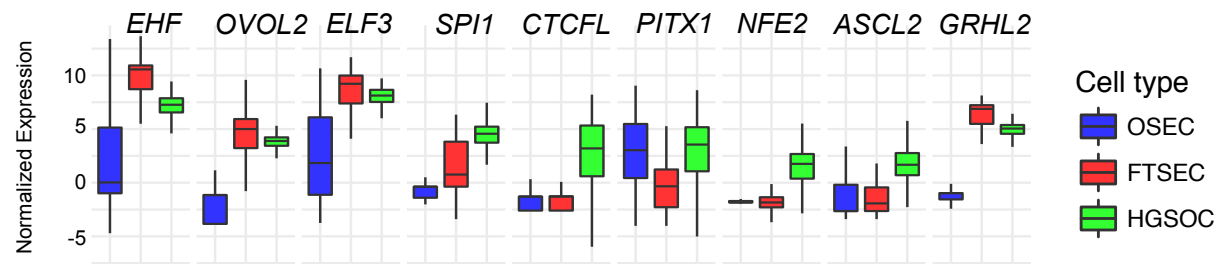

b Binding at DEGs in HGSOC versus FTSEC (162 genes)

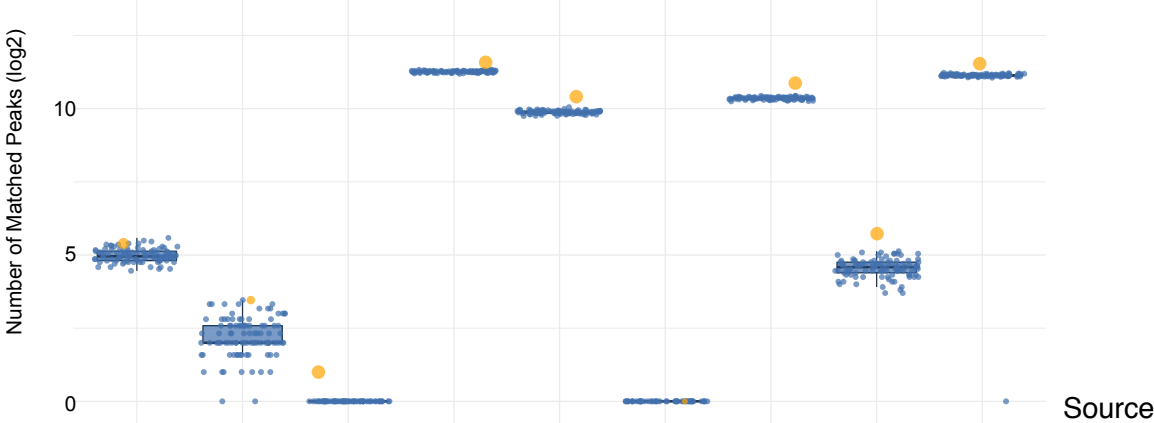

C Binding at DEGs in HGSOC versus OSEC (419 genes)

- Random

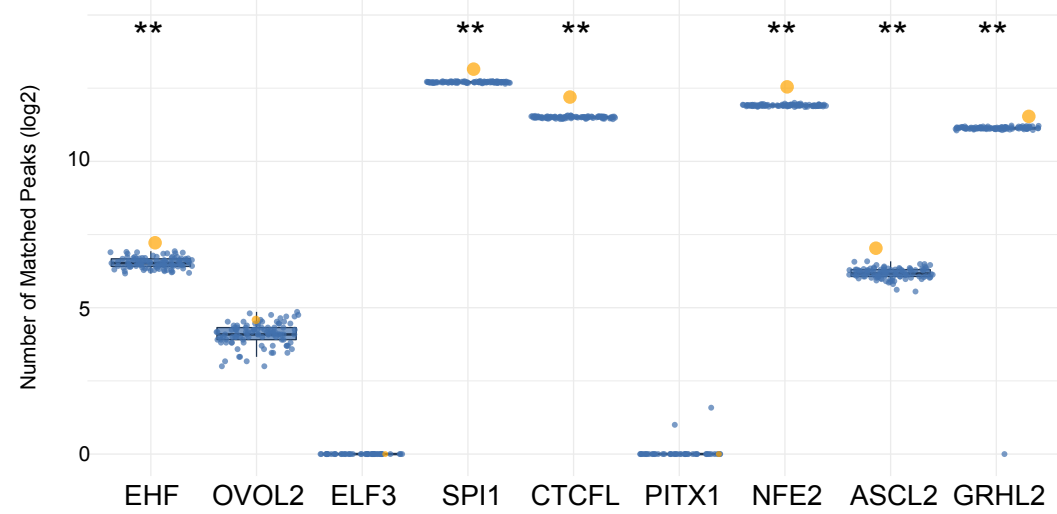

